\title{
Current status of intralesional agents in treatment of malignant melanoma
}

\author{
Misam Zawit ${ }^{1 \# \wedge}$, Umang Swami ${ }^{2 *}$, Hassan Awada ${ }^{1}$, Joyce Arnouk ${ }^{3}$, Mohammed Milhem $^{3}$, \\ Yousef Zakharia ${ }^{3}$
}

${ }^{1}$ Department of Translational Hematology and Oncology Research, Taussig Cancer Institute, Cleveland Clinic Foundation, Cleveland, OH, USA; ${ }^{2}$ Department of Internal Medicine, Huntsman Cancer Institute, University of Utah, Salt Lake City, UT, USA; ${ }^{3}$ Division of Hematology, Oncology and Blood and Marrow Transplantation and the Holden Comprehensive Cancer Center, University of Iowa Hospitals and Clinics, Iowa City, IA, USA

Contributions: (I) Conception and design: M Zawit, U Swami, M Milhem, Y Zakharia; (II) Administrative support: All authors; (III) Provision of study materials or patients: All authors; (IV) Collection and assembly of data: M Zawit, U Swami, M Milhem, Y Zakharia; (V) Data analysis and interpretation: M Zawit, U Swami, M Milhem, Y Zakharia; (VI) Manuscript writing: All authors; (VII) Final approval of manuscript: All authors.

\#These authors contributed equally to this work.

Correspondence to: Yousef Zakharia, MD. Division of Hematology, Oncology and Blood and Marrow Transplantation and the Holden Comprehensive Cancer Center, University of Iowa Hospitals and Clinics, 200 Hawkins Dr., Iowa City, IA 52242, USA. Email: yousef-zakharia@uiowa.edu.

\begin{abstract}
Prognosis of metastatic melanoma has undergone substantial improvement with the discovery of checkpoint inhibitors. Immunotherapies and targeted therapies have improved the median overall survival (OS) of metastatic melanoma from 6 months to more than 3 years. However, still about half of the patients die due to uncontrolled disease. Therefore, multiple strategies are currently being investigated to improve outcomes. One such strategy is intralesional/intratumoral (IT) therapies which can either directly kill the tumor cells or make the tumor more immunogenic to be recognized by the immune system. Talimogene laherparepvec (T-VEC), an oncolytic virus, is the first FDA approved IT therapy. This review focuses on the current status of IT agents currently under clinical trials in melanoma. Reviewed therapies include T-VEC, T-VEC with immune checkpoint inhibitors including ipilimumab and pembrolizumab or other agents, RP1, OrienX010, Canerpaturev (C-REV, HF10), CAVATAK (coxsackievirus A21, CVA21) alone or in combination with checkpoint inhibitors, oncolytic polio/rhinovirus recombinant (PVSRIPO), MAGE-A3-expressing MG1 Maraba virus, VSV-IFNbetaTYRP1, suicide gene therapy, ONCOS-102, OBP-301 (Telomelysin), Stimulation of Interferon Genes Pathway (STING agonists) including DMXAA, MIW815 (ADU-S100) and MK-1454, PV-10, toll-like receptors (TLRs) agonists including TLR-9 agonists (SD-101, CMP-001, IMO-2125 or tilsotolimod, AST-008 or cavrotolimod, MGN1703 or lefitolimod), CV8102, NKTR-262 plus NKTR-214, LHC165, G100, intralesional interleukin-2, Daromun (L19IL2 plus L19TNF), Hiltonol (polyICLC), electroporation including calcium electroporation and plasmid interleukin-12 electroporation (pIL-12 EP), IT ipilimumab, INT230-6 (cisplatin and vinblastine with an amphiphilic penetration enhancer), TTI621 (SIRP $\alpha$ Fc), CD-40 agonistic antibodies (ABBV-927 and APX005M), antimicrobial peptide LL37 and other miscellaneous agents.
\end{abstract}

Keywords: Melanoma; intralesional; intratumoral; talimogene laherparepvec (T-VEC)

Submitted Jan 31, 2021. Accepted for publication Apr 16, 2021.

doi: $10.21037 / \mathrm{atm}-21-491$

View this article at: http://dx.doi.org/10.21037/atm-21-491

^ ORCID: Misam Zawit, 0000-0003-2991-4386; Umang Swami, 0000-0003-3518-0411. 


\section{Introduction}

Metastatic melanoma is one of the biggest success stories in the fight against cancer over the last decade. Before the advent of checkpoint inhibitors (CPI) and BRAF/MEK inhibitors, the median overall survival (OS) for patients with metastatic melanoma was a meager 6 months which has now improved to more than 3 years $(1,2)$. However, many patients still progress on currently approved therapies, and multiple strategies are being investigated to improve outcomes for these refractory patients. One of these strategies involves intralesional/intratumoral (IT) injections. Metastatic melanoma is a unique tumor which apart from having a high mutational burden (3) and targetable mutations in half of the patients (4), has many times palpable lesions. This has provided opportunities for developing many IT therapies in effort to make it more susceptible to CPI. In this review, we summarize therapies that are currently being investigated as IT in advanced melanoma. Figure 1 summarizes the mechanism of action of selected agents.

\section{Materials and methods}

A literature search traversed PubMed, Medline, and the American Society of Clinical Oncology abstracts between 1970 and May 2019. Search terms included melanoma, malignant melanoma, and metastatic melanoma, in combination with the following terms/ agent names [intralesional, intratumoral, oncolytic virus, oncolytic virotherapy, talimogene laherparepvec, T-VEC, intralesional T-VEC and pembrolizumab, HF10, CAVATAK, coxsackievirus A21, CVA21, oncolytic polio/ rhinovirus recombinant, PVSRIPO, MAGE-A3, Maraba virus, VSV-IFNbetaTYRP1, ONCOS-102, OBP-301, Telomelysin, stimulation of interferon genes pathway, STING agonists, DMXAA, MIW815 (ADU-S100), MK-1454, Rose Bengal, PV-10, toll-like receptors (TLRs) agonists, TLR-9 agonists, SD-101, CMP001, IMO-2125, Tilsotolimod, AST-008, MGN1703, CV8102, NKTR-262 plus NKTR-214, LHC165, G100, intralesional interleukin-2, IL-2, Daromun, L19IL2 plus L19TNF, Hiltonol, poly-ICLC, electroporation, calcium electroporation, plasmid interleukin-12 electroporation, pIL-12 EP, IT ipilimumab, INT230-6 (cisplatin and vinblastine with an amphiphilic penetration enhancer), TTI-621 (SIRP $\alpha$ Fc protein), Toca 511 (vocimagene amiretrorepvec) and Toca FC (5-fluorocytosine), CD-40 agonistic antibodies, ABBV-927, LL37].

\section{Intralesional therapies}

\section{Oncolytic viruses}

The idea of oncolytic virotherapy arose more than a century ago when influenza was found to cause the disappearance of excess leukocytes in a patient with acute leukemia (5). Thereafter animal studies since the 1920s and human trials since the 1960s have been conducted in various cancer types with limited success (6). Some viruses such as mumps and Moloney leukemia virus have a natural predilection towards tumor cells while others such as adenovirus (Ad), vesicular stomatitis and herpes simplex viruses have been genetically modified to be used as therapeutic agents (7). The sought after effect of virotherapy includes selective infection of tumor cells, followed by resultant direct killing by "viral debulking" or host immune response against virus-infected cells and subsequent tumor cell killing by antitumor immune response (7).

\section{Talimogene laherparepvec (T-VEC)}

T-VEC is a live attenuated herpes simplex virus type 1 (HSV1), developed from genetically modified JS-1 strain; by disabling the gene ICP34.5; to decrease neurovirulence and boost predilection to tumor cells, and deletion of ICP47 gene to promote immune response. Furthermore, T-VEC is engineered to express human granulocyte-macrophage colony-stimulating factor (GM-CSF) which leads to dendritic cell recruitment (8). T-VEC (OncoVEXGM-CSF, Imlygic $\left.^{\circledR}\right)$ is the first oncolytic viral therapy to be approved by the Food and Drug Administration (FDA) in 2015 for local treatment of unresectable, cutaneous, subcutaneous, and nodal lesions in patients with melanoma recurrent after initial surgery (8).

In a phase I trial, 30 patients with cutaneous or subcutaneous metastatic solid tumors (breast, colorectal, melanoma, and head and neck cancer) were treated with variable doses of T-VEC (9). Twenty-six patients were evaluable of whom 6 patients were noted to have flattened lesions after injection (2 responded in noninjected lesions). Side effects mainly included B symptoms, grade 1 febrile illness, nausea, vomiting, fatigue, and low-grade anorexia (9). In the subsequent phase II trial of 50 patients with stage IIIc and IV melanoma, the overall response rate (ORR) was $26 \%$ (13/50 patients) of which eight patients achieved a complete response (CR). The response was noted at injected and distal lesions and lasted for 7-31 months. OS was $58 \%$ at 1 year and $52 \%$ at 24 months (10).

These encouraging results lead to a phase III trial of 


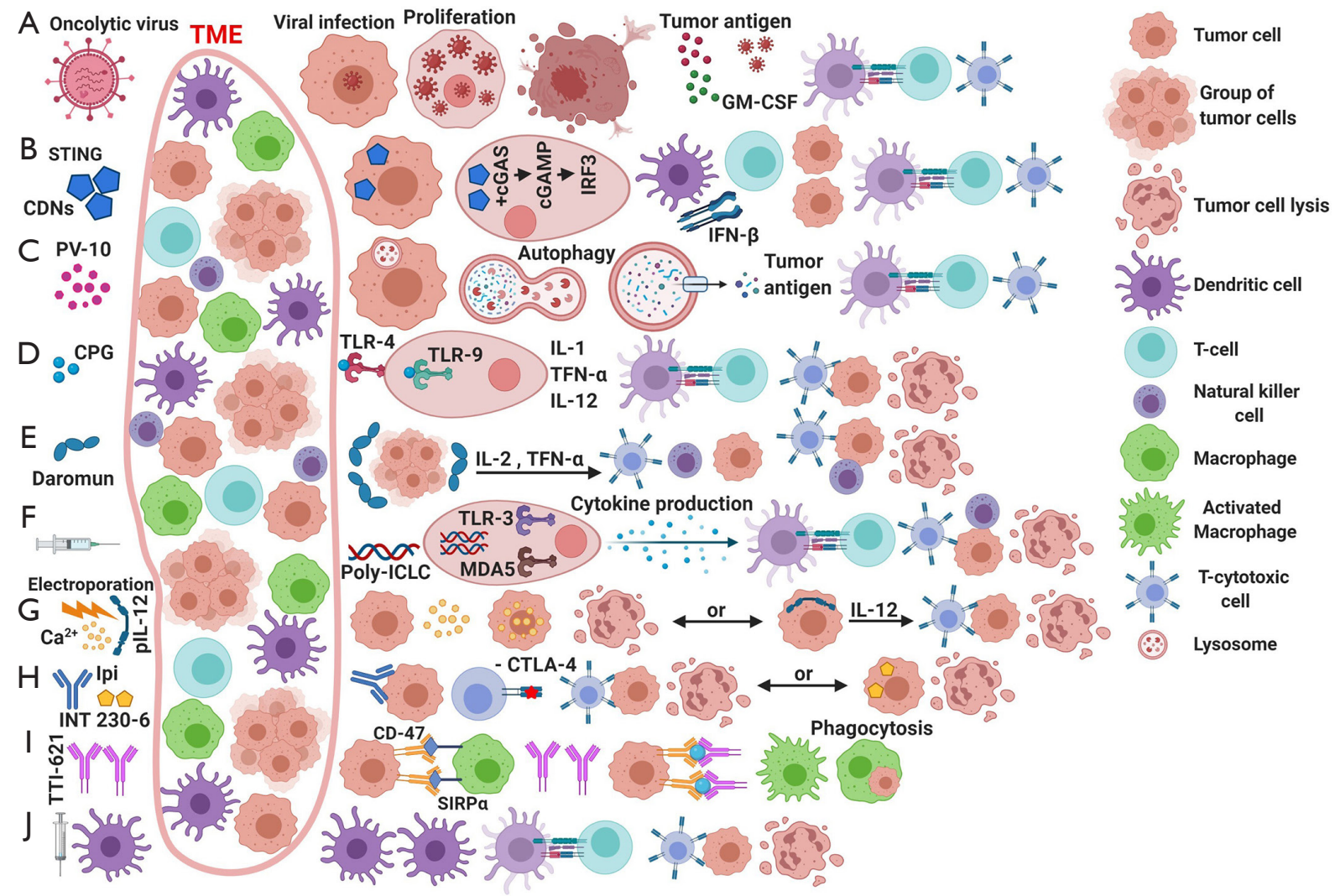

Figure 1 Mechanism of action of different intralesional therapeutic regimens. (A) Oncolytic viruses pathway; T-VEC penetrate the cells, followed by virus proliferation and resultant cell lysis. Tumor antigens are made available through cytolysis, these antigens will be presented by $\mathrm{DC}$ and macrophages to cytotoxic $\mathrm{CD}^{+} \mathrm{T}$ lymphocytes, resulted in turn in activation of immune response against tumor cells. (B) STimulator of INterferon Genes (STING) pathway agonists; CDNs recognized by cGAS, resulting in the production of cGAMP, which in turn binds to STING molecule which will undergo a conformational change, subsequently resulting in a series of molecular interactions in the pathway of INF- $\beta$ production. Increased INF- $\beta$ expression will stimulate antigen-presenting cells (APCs) and increase the presence of infiltrating T-lymphocytes. (C) Rose Bengal-10 (PV-10). PV-10 preferentially absorbed by tumor cells to a larger extent in comparison to normal cells. Accumulating in the lysosomes, will then result in cell necrosis, release of tumor antigens, resulting in tumor specific immune response. (D) Toll-like receptors (TLRs) agonists: activating TLRs by CpG molecules will lead to stimulation of nuclear factor kappa-light-chain-enhancer of activated B (NF-кB) pathway, leading to increase production of several chemokines and cytokines (e.g., IL1, IL-12 and TNF- $\alpha$ ) upregulating antigen presenting cells and increasing lymphocytes infiltration. (E) Intralesional interleukin-2 (IL2) or Daromun administration: IL-2 potentiate T-helper cells differentiation and proliferation, cytolytic CD $8^{+} \mathrm{T}$-cells and natural killer (NK) cells function, and also plays an important part in other cytokines production such as IL-9; (F) Intratumoral vaccines: Hiltonol (polyICLC) is able to produce an antitumor response by activating TLR-3 and MDA5, subsequently activating APCs, NK cells, T-cells, and upregulate the production of cytokines and chemokines. (G) Electroporation: by using short electrical pulses administered directly to the tumor, resulting in an increased permeability of tumor-cell walls to certain molecules (e.g., $\mathrm{Ca}^{2+}$ or pIL-12) that under normal circumstances are unable to penetrate the cell membrane. markedly increased intracellular calcium concentration results in tumor-cell necrosis due to sudden exhaustion of adenosine triphosphate (ATP) reserve. Or using the electroporation technology to introduce plasmid DNA that carries IL-12 gene, capable of transcribing RNA into tumor cells and ultimately increase IL-12 production resulting in an increased local immune response. $(\mathrm{H})$ Intratumoral immunotherapy/chemotherapy; ipilimumab a human antibody directed against cytotoxic $\mathrm{T}$ lymphocyte associated antigen-4 receptor (CTLA-4), inhibiting CTLA-4 receptor will lead to an augmented local immune response by promoting T-cell activation and increase IL-2 production. Injected INT230-6 results in increased intracellular concentration of the combined chemotherapeutic agents (cisplatin and vinblastine) resulting in tumor cellular death, along with an increased concentration of immune cells (e.g., DC and T-cells) in the tumor microenvironment. (I) Other intratumoral agents: TTI-621 (SIRP $\alpha$ Fc) protein: TTI-621 is recombinant 
human protein, that contains the N-terminal V domain of human SIRP $\alpha$ attached to an Fc region of human immunoglobulin G1 (IgG1). Administration of this agent will inhibit the CD47-SIRP $\alpha$ signal, resulting in an unblocked phagocytic function of the host macrophages against tumor cells. (J) Miscellaneous: dendritic cell therapy after cryotherapy in combination with pembrolizumab: injecting autologous vaccine using patient's mature dendritic cells plus tumor proteins after undergoing lesion cryotherapy. Preceded by IV pembrolizumab. TME, tumor microenvironment; T-VEC, talimogene laherparepvec; GM-CSF, granulocyte monocyte-colony stimulating factor; CDN, cyclic dinucleotide; cGAS, cyclic GMP-AMP synthetase; cGAMP, cyclic GMP-AMP; IRF3, interferon regulatory transcription 3; PV-10, Rose Bengal-10; TLR, toll-like receptor; CpG, oligonucleotide with cytidine-phospho-guanosine patterns; NF- $\mathrm{B}$, nuclear Factor kappalight-chain-enhancer of activated B pathway; IL-1, interleukin-1; IL-12, interleukin-12; TNF- $\alpha$, tumor necrosis factor-alpha; Daromun (L19IL2 + L19TNF), interleukin-2 attached to L19 antibody fragment + tumor necrosis factor attached to L19 antibody fragment; polyICLC, a manufactured double-stranded RNA of polyinosinic-polycytidylic acid-poly-l-lysine carboxymethylcellulose; MDA5, melanoma differentiation associated protein 5; pIL-12, plasmid interleukin-12; Ipi, ipilimumab; INT230-6, cisplatin and vinblastine with an amphiphilic penetration enhancer; $\operatorname{SIRP} \alpha$, signal-regulatory protein alpha; CD47, cluster of differentiation 47.

436 patients with stage IIIB-IV malignant melanoma not amenable for surgical intervention. Patients were randomized in a $2: 1$ ratio to either IT T-VEC or subcutaneous GM-CSF respectively. The primary endpoint of durable response rate (DRR) (defined as ORR lasting continuously $\geq 6$ months) was significantly higher in the T-VEC arm as compared to the GM-CSF arm (16.3 vs. $2.1 \%$; odds ratio, $8.9 ; \mathrm{P}<0.001)$. The secondary endpoint of ORR favored experimental arm $(26.4 \%$ with T-VEC $v s$. $5.7 \%$ in GM-CSF, $\mathrm{P}<0.001)$. There was non-statistically significant trend for improved median OS in T-VEC arm (23.3 months) vs. GM-CSF (18.9 months) (HR, 0.79; 95\% CI, 0.62-1.00; $\mathrm{P}=0.051$ ). Adverse events (AEs) were mild in general and more common in the T-VEC arm. The most common any grade AEs noticed with T-VEC were fatigue $(50.3 \%)$, followed by chills (48.6\%), pyrexia $(42.8 \%)$, and nausea $(35.6 \%)$. Injection site erythema was more common in the GM-CSF arm (26\% vs. 5.1\%). Incidence of grade 3 or 4 treatment-related AEs was more in the T-VEC arm as compared to the GM-CSF arm (11\% vs. 5\%) with no treatment-related grade 5 events (11).

\section{T-VEC with other agents}

\section{Intralesional T-VEC with ipilimumab}

IT T-VEC in combination with ipilimumab (anti-CTLA-4 antibody) has been tested in patients with advanced-stage melanoma (IIIB-IV). In a phase $1 \mathrm{~b}$ trial (12), 19 patients were enrolled. No dose limiting toxicities (DLTs) were observed. Five patients (26.3\%) reported grade 3 or higher AEs of which in three patients AEs were related to T-VEC and in four to ipilimumab. Elevated lipase and amylase were the only grade $4 \mathrm{AE}$ and were related to ipilimumab. ORR was $50 \%$. A DRR (more than 6 months response) was achieved in $44 \%$ of patients. Eighteen months of progression-free survival (PFS) and OS were 50\% and 67\% respectively (12). In a phase II trial, 198 patients with stage IIIB-IV melanoma were randomly allocated to T-VEC plus ipilimumab versus ipilimumab alone. The study met its primary endpoint of significantly higher ORR in the combination arm as compared to the ipilimumab arm $(39 \%$ vs. $18 \%$; odds ratio, $2.9 ; 95 \% \mathrm{CI}, 1.5-5.5 ; \mathrm{P}=0.002)$. As compared to the ipilimumab alone arm, more patients in the combination arm experienced all-grade (98\% vs. 95\%) and grade $\geq 3$ AEs (45\% vs. 35\%) (13).

\section{Intralesional T-VEC with pembrolizumab}

In a phase Ib study of T-VEC with pembrolizumab (antiPD-1 antibody) in patients with advanced-stage (IIIbIV) melanoma, 21 patients were enrolled. There was a $62 \%$ ORR (33\% CR) with no DLTs. AEs that can be attributed to either agent, were fatigue (62\%), chills (48\%), and fever (43\%). Serious side effects included grade 3 aseptic meningitis, grade 3 autoimmune hepatitis, grade 4 pneumonitis, and grade 1 cytokine-release syndrome required inpatient hospital stay (1 patient each) (14).

In another ongoing phase III clinical trial, patients $(\mathrm{n}=713)$ with stage IIIB to IVM1c melanoma are treated with pembrolizumab with or without T-VEC (MASTERKEY-265/KEYNOTE-034, NCT02263508). The same combination is being evaluated as neoadjuvant therapy in patients with stage III resectable, cutaneous melanoma with clinically palpable lymph node metastases (NCT03842943).

Multiple other trials are ongoing combining T-VEC with various agents such as chemotherapy melphalan administered by isolated limb perfusion (ILP) (NCT03555032), with dabrafenib and trametinib in BRAF-mutated melanoma (NCT03088176) and IT autologous CD1c (BDCA-1) positive myeloid dendritic cells (NCT03747744). 
A phase II randomized, trial (NCT02211131) is evaluating the efficacy and safety of surgery with or without T-VEC as neoadjuvant therapy for resectable stages IIIBIVM1 a melanoma. The primary goal is the evaluation of recurrence-free survival (RFS). Patients are randomized in a 1:1 ratio to both study arms, with arm 1 receiving 6 doses of T-VEC prior to surgery while arm 2 undergoing surgical resection alone. Interim analysis of 150 randomized patients showed a $15.8 \%$ pathological CR and $42.1 \%$ negative resection margin (R0) in arm 1, while $\mathrm{R} 0$ in arm 2 was $37.8 \%$. Primary outcome analysis of RFS is pending (15).

\section{Other HSV based agents}

\section{RP1}

RP1 is a genetically engineered, HSV1 encoded with GM-CSF, which can be intratumorally administered. It is undergoing a phase I/II clinical trial (NCT03767348) to determine its safety, tolerability, along with effectiveness as a monotherapy and in combination with nivolumab, in patients with advanced solid tumors including melanoma.

\section{OrienX010}

OrienX010 is another genetically modified HSV1 oncolytic virus similar to T-VEC but made from CL1 strain (16). It is undergoing a phase 1-c study (NCT03048253) in patients with melanoma metastatic to the liver who have been previously treated. The primary endpoint is the safety of OrienX010, while the secondary endpoints are ORR, disease control rate (DCR), and PFS time. Available data for 15 patients showed only grade $1 / 2$ AEs including flulike symptoms and pain at the injection site, as well as nausea/vomiting, hepatotoxicity, and leukopenia. Results of 12 evaluable patients after a median follow-up time of 6 months showed an $8.3 \%$ ORR, $41.7 \%$ DCR [ 1 partial response (PR), 4 stable disease (SD)], and a median PFS of 13.3 weeks. Median OS has not reached (17).

\section{Canerpaturev (C-REV, formerly HF10)}

$\mathrm{C}-\mathrm{REV}$ is a non-engineered, naturally occurring HF10 strain of HSV1 virus, with unique deletions and insertions in its genetic structure which gives it the ability to preferentially infect cancer cells, and proliferate at a high rate, resulting in cytolytic effect (18). The inherently occurring genomic deletions, cause certain genes [i.e., UL43, UL49.5, UL55, UL56, and latency-associated transcript (LAT)] to be silenced, at the same time, insertions lead to increased translation of $U L 53$ and $U L 54$ genes. These changes lead to decreased virulence, enhanced replication in tumor cells, and augmented immune response. Most notable, the HF10 virus loses its neurovirulence and latent infection properties because of UL56 and LATs genes deletions (18).

In a phase I trial assessing the safety of IT administered HF10, 26 patients with malignant melanoma and other refractory and superficial tumors were enrolled. Of the 24 patients who received treatment, 18 developed AEs of which 6 were related to HF10. These included chills (2 patients), discoloration, swelling, and pain at the injection site (1 patient), malaise (1 patient), pruritus (1 patient), and hypotension (1 patient). One patient developed ulceration of tumor sites at both injected and non-injected lesions after one injection while normal tissue did not develop any ulceration (19).

In a phase II study of combination therapy of IT HF10 and ipilimumab in patients with stage IIIb-IV melanoma, 46 patients were enrolled. The primary endpoint was the best ORR at 24 weeks. No DLTs or severe AEs of HF10 were reported. Overall, $37 \%$ of patients developed grade 3 or higher AEs, most of which were attributed to ipilimumab. Three grade 3 or higher AEs were attributed to HF10 including left groin pain, lymphedema, and thromboembolic event (one patient), hypoglycemia (one patient), and diarrhea (one patient). Out of the 44 evaluable patients, the best ORR was $41 \%$ and the disease stability rate was $68 \%$ at 24 weeks. At the time of data cut-off, the median PFS was 19 months and the median OS was 26 months $(20,21)$.

In another phase I/II study of HF10 with or without ipilimumab in Japanese patients with advanced solid tumors, results showed that IT HF10 can enhance the immune response to ipilimumab (22). Another phase II trial (NCT03259425) is currently examining the safety and efficacy of the neoadjuvant nivolumab with IT HF10 in patients with resectable stages IIIB-IV melanoma.

\section{CAVATAK [coxsackievirus A21 (CVA21)]}

CVA21, is an enterovirus of the Picornaviridae family. In humans, it causes the common cold. Due to its inherent predilection to cancer cells in its natural form, CVA21 has been commercially fashioned from Kuykendall strain as CAVATAK (23). CVA21 infectivity has been found to be in part due to its binding ability to intercellular adhesion molecule-1 (ICAM-1), and to a lesser extent due to its binding to the decay-accelerating factor (DAF) molecules on the cell surface. The virus is subsequently entrapped 
inside the cell (23). ICAM-1 and DAF receptors are abundantly expressed on melanoma cells making melanoma a potential target for oncolytic effect of CAV21 (23).

IT CVA2 1 was well tolerated in a phase 1 study that enlisted 9 patients with stage IV melanoma and effectively reduced the size of injected lesions in 5 out of 9 patients. A high level of serum GM-CSF was noted in 2 of the 5 patients who experienced lesion size reduction, highlighting the possibility of systematic immune-mediated anti-tumor effect of CAV21 (24). Phase II CALM (CAVATAK in latestage melanoma) trial enrolled 57 patients with stages IIIIV unresectable melanoma. The primary endpoint was more than 9 of 54 evaluable patients achieving immunerelated PFS (irPFS) at 6 months. The study accomplished its primary goal, with $38.6 \%(\mathrm{n}=21)$ of evaluable patients demonstrating irPFS at 6 months. The immune-related Response Evaluation Criteria in Solid Tumors (irRECIST) ORR was $28.1 \%$ (16 of 57 ) with $\geq 6$ months DRR of $19.3 \%$ (11 of 57 patients). The 1 -year survival rate was $75.4 \%$ (43 of 57 patients). Consistent with other oncolytic viral therapies, AEs of CAVATAK were grade I chill, fatigue, fever, and local injection site reactions. No grade 3 or 4 AEs were reported (25). In the extension cohort, sequential tumor biopsies of both injected and non-injected lesions were done to evaluate viral replication and immune activation within the tumor microenvironment. Results were significant for reconstitution of immune cell infiltrates in lesions resistant to immune-checkpoint blockade along with up-regulation of various immune-related genes such as Th1-gene shift and increases in interferon-induced genes (26). This has provided the rationale for CVA21 combination studies with CPI.

CVA21 was combined with ipilimumab in phase Ib, MITCI trial (NCT02307149). Available results from 23 patients with unresectable stage IIIC-IV melanoma, showed that the treatment is well-tolerated with no DLT and only one grade $\geq 3 \mathrm{AE}$ of ipilimumab-related fatigue. Of the evaluable 18 patients, the confirmed ORR was 50\% (9/18) and DCR (CR + PR + SD) of 78\% [14/18]. Exploratory analysis revealed an increase in the percentage of activated $\mathrm{CD}^{+}$and $\mathrm{CD}^{+}{ }^{+} \mathrm{T}$-cells with effector and memory phenotypes in the peripheral blood of patients treated with combination therapy (27).

CVA21 was also investigated in combination with pembrolizumab in a phase 1b study (VLA-011 CAPRA, NCT02565992) in stage IIIC-IVM1c melanoma patients. The primary endpoint was safety and tolerability of the combination. Available results from 14 patients did not show any DLTs or unanticipated AEs. Of the 11 evaluable patients, ORR was 73\% [8/11] and overall DCR (CR + PR + SD) was $91 \%$ [10/11] (28).

\section{Oncolytic polio/rhinovirus recombinant (PVSRIPO)}

PVSRIPO is a recombinant, live-attenuated, oncolytic hybrid of poliovirus Sabin type 1 (PV1) and human rhinovirus type 2 (HRV2). In PVSRIPO, the internal ribosomal entry site (IRES) of PV1 is replaced with that of HRV2 resulting in a marked decrease in PV1 virulence against neuronal cells. PV1 receptor, CD155 is ubiquitously expressed on solid tumor cells, particularly melanoma cells which accounts for PVSRIPO tropism and selectivity which along with IRES-mediated translation and viral replication gives rise to sustained type 1 interferon response which is vital for a successful antitumor immune response (29). PVSRIPO has shown promising safety results in recurrent glioblastoma (29). Currently, it is undergoing safety evaluation in melanoma patients in a phase I trial (NCT03712358). The study aims to enlist up to 9 participants, with stages IIIB-IV. PVSRIPO will be administered intratumorally into $1-3$ cutaneous or subcutaneous lesions.

\section{MAGE-A3-expressing MG1 Maraba virus}

Maraba virus is an oncolytic virus belonging to the Rhabdoviridae family and Vesiculovirus genus (30). It retains its ability to infect cells, by utilizing the low-density lipoprotein receptor (LDLR) expressed on the target cell to facilitate cellular penetration. To further enhance its potential and selectivity toward malignant cells, the Maraba virus was genetically modified by the introduction of twopoint mutations altering the $M$ and $G$ proteins, creating the MG1 strain. MG1 virus was further strengthened by adding transgenes overexpressing tumor antigens to create an "oncolytic vaccine" (30). An inert Ad vector was also modified by incorporating transgenes overexpressing tumor antigens. Coupled together Ad and MG1 viral vectors encoding human melanoma-associated antigen-A3 (MAGE-A3), are a potent stimulator of the adaptive immune system, specifically against malignant cells (30).

A phase Ib clinical trial (NCT03773744) is evaluating the safety and tolerability of intravenous (IV) or IT MG1MAGE with Ad-MAGEA3 in patients with metastatic melanoma or previously treated cutaneous squamous cell carcinoma patients. The study has two arms. In arm 1, participants will receive low-dose cyclophosphamide, and intramuscular (IM) Ad-MAGEA3 prime, followed 
by IV MG1-MAGEA3 and IV pembrolizumab. In arm 2 participants will receive IM Ad-MAGEA3 injection as a prime, and IV MG1-MAGEA3, followed by IT MG1MAGEA3 and IV pembrolizumab.

\section{Recombinant vesicular stomatitis virus-expressing interferon-beta and tyrosinase related protein 1 (VSV- IFNbetaTYRP1)}

VSV-IFNbetaTYRP1, is an attenuated, recombinant vesicular stomatitis virus in which human genes responsible for interferon-beta (INF- $\beta$ ) and tyrosinase-related protein 1 (TYRP1) have been added (31). The safety and efficacy of IV and IT VSV-INFbetaTYRP1 is being evaluated in a phase I clinical trial (NCT03865212) in patients with stages III/IV unresectable cutaneous and metastatic ocular melanoma.

\section{Suicide gene therapy}

Suicide gene therapy entails the use of therapeutic transgenes which can encode genes with the ability to convert a non-toxic prodrug into a cytotoxic agent or which can express toxic gene expression product (32). NCT03338777 was a phase I study which evaluated lipoplexes carrying the suicide gene system herpes simplex virus thymidine kinase (HSVtk)/pro-drug ganciclovir (33) and subcutaneous vaccine produced with formalized allogeneic tumor extracts and lipoplexes carrying human interleukin-2 (IL-2) and human GM-CSF genes. The study was terminated after the enrollment of 4 patients.

$\mathrm{ADV} / \mathrm{HSV}$-tk is another suicide gene therapy, consisting of Ad vector engineered expression of herpes simplex virus thymidine kinase (ADV/HSV-tk) gene therapy administered intratumorally along with synthetic acyclic guanosine analogue such as oral valacyclovir, acyclovir, or ganciclovir (33). A window of opportunity, now terminated phase II trial (NCT02831933) aimed to evaluate the efficacy and safety of ADV/HSV-tk and stereotactic body radiation therapy to patients with metastatic non-small cell lung cancer and metastatic uveal melanoma before initiating nivolumab.

Toca 511 and Toca FC is a combination of gene therapy and prodrug $(34,35)$. Toca 511 (vocimagene amiretrorepvec) is a genetically modified, replicating, retroviral vector that contains a yeast gene responsible for cytosine deaminase expression that converts 5-fluorocytosine (an antifungal agent) to 5-fluorouracil (5-FU), a known chemotherapy $(34,35)$. Toca FC is an extended-release formulation of antifungal agent 5-fluorocytosine. Toca 511, being a retroviral replicating vector selectively infects and replicates in tumor cells, because of the defective antiviral mechanisms in malignant cells and tumor microenvironment. This leads to a higher concentration of 5-FU inside the tumor as compared to systematically administered 5-FU (34,35). In a now terminated phase I study (NCT02576665) Toca511 was evaluated in combination with Toca $\mathrm{FC}$ in patients with different malignancies including melanoma.

\section{ONCOS-102}

ONCOS-102 (Ad5/3-A24-GM-CSF) is an oncolytic, genetically modified Ad that expresses GM-CSF. Infection of tumor cells occurs through binding to desmoglein 2 receptor commonly expressed on tumor cell surfaces due to chimeric $5 / 3$ capsid which consists of fiber knob derived from Ad serotype 3. Once infection occurs, ONCOS-102 replicates in tumor cells as a result of the 24 bp deletion in the $\mathrm{Rb}$ binding site of the $E 1 A$ gene which limits its replication to cells with $\mathrm{p} 16-\mathrm{Rb}$ pathway defects, which are found in a majority of malignant tumors (36). As in T-VEC, local production of GMCSF increases infiltration of NK cells and $\mathrm{CD} 8^{+} \mathrm{T}$-cells in the injected tumor microenvironment leading to antitumor immune responses (36). NCT03003676 Is a phase I, multicenter study recruiting patients with advanced or unresectable, PD-1 refractory melanoma. This two-part study aims to determine the safety of IT ONCOS-102 and IV pembrolizumab after cyclophosphamide priming in different sequences.

\section{OBP-301 (Telomelysin)}

Telomelysin (OBP-301) is a genetically engineered oncolytic Ad serotype-5-based Ad construct which (1) has been modified by the addition of human telomerase reverse transcriptase gene (bTERT) promoter which encodes for catalytic protein subunit of telomerase, (2) replacement of the normal transcriptional element of viral $E 1 B$ gene by an IRES sequence to minimize "leakiness" and (3) retains fully functional viral E3 region (37). In a phase I study 16 patients with advanced solid tumors including malignant melanoma were administered IT Telomelysin. The majority of AEs were grade 1/2, including pain, swelling at the injection site, chills, and fever. One patient had a PR and seven had SD (37). A phase II study (NCT03190824) is currently assessing the efficacy of OBP-301 in patients with 
metastatic melanoma.

\section{Stimulation of interferon genes patbway (STING agonists)}

The cyclic guanosine monophosphate-adenosine monophosphate synthase-stimulator of interferon genes (cGAS-STING) pathway play role in tumor immunity (38). DNA from any source, foreign or self can enter cytosol and activate cyclic GMP-AMP synthetase (cGAS) to generate cyclic GMP-AMP (cGAMP) which subsequently binds to the endoplasmic reticulum (ER)-localized adaptor protein STING. Apart from DNA, the STING pathway can also be activated by viral liposomes, cyclic dinucleotides, and ER stress. Once cGAMP bind to the STING molecule, the latter undergoes a conformational change and traffics from ER to Golgi where it recruits various kinases which subsequently phosphorylate transcription factors leading to transcription of genes encoding type 1 interferons such as interferon- $\beta$ and pro-inflammatory cytokines such as IL-6 and tumor necrosis factor (38). STING signaling can also activate microtubule-associated protein $1 \mathrm{~A} / 1 \mathrm{~B}$-light chain 3-mediated autophagy, apoptosis, and necroptosis (38). Increased interferon- $\beta$ and pro-inflammatory cytokine signaling promotes the immune response by enhancing cross-presentation and antitumor activity by stimulating antigen-presenting cells (APCs) such as dendritic cells and enhancing the cytotoxic activity of infiltrating T-lymphocytes, facilitating the transition to a robust adaptive immune response (39).

The first described STING pathway agonist in medical literature was 5,6-dimethylxanthenone-4-acetic acid (DMXAA), which initially showed anti-tumor potentials in mouse models. However, no therapeutic benefits were noted when studied in clinical trials. Further understanding of the molecular mechanism behind such failure, which was related to different structures between mouse versus human STING molecule, opened the door to revisit STING pathway agonists as a novel therapeutic approach in cancer patients (40).

Two STING Agonists, MIW815 (ADU-S100) and MK1454 are currently under study in clinical trials as IT agents in solid tumors (including melanoma) and lymphoma. MIW815 is being investigated in a phase $\mathrm{Ib}$, multicenter, open-label study (NCT03172936), in combination with PDR-001, an anti-PD1 antibody. NCT02675439 is a phase I, open-label, multicenter study of ADU-S100 with or without ipilimumab. Both studies are evaluating the safety, tolerability, pharmacological properties, and antitumor activity of the combination therapy. Another phase I study NCT03010176 is evaluating ITMK-1454 as a single agent or in combination with pembrolizumab. Preliminary results showed encouraging activity and safety profile. PRs in head and neck squamous cell carcinoma, triple-negative breast cancer, and anaplastic thyroid carcinoma were observed (41).

\section{PV-10}

Rose Bengal is a xanthene dye, that has been historically used intravenously to assess liver function, and later used in ophthalmology clinic as an eye dye (42). PV-10 is a $10 \%(\mathrm{w} / \mathrm{v})$ solution of Rose Bengal in saline, manufactured for IT administration (42). Upon IT injection, PV-10 is preferentially taken by lysosomes of tumor cells as compared to normal cells resulting in tumor necrosis. It subsequently leads to the release of tumor antigens translating into tumor-specific immune response (43). In animal models, IT PV-10 administration has shown to cause tumor regression in both injected and noninjected lesions, supporting the notion that PV-10 therapy results in systemic tumor-specific immune response (42).

In a phase I clinical trial, 11 participants with stage III melanoma, received PV-10. A total of 26 lesions were injected and 28 distant noninjected lesions were observed. Treatment was well tolerated with no DLTs. Predominant side effects were local AEs including injection site pain, local inflammation, and local pruritus. Photosensitivity involving the treated extremity was reported in one patient, while insomnia was the only reported systemic AE due to injection site pain in one patient. Per RECIST criteria, the ORR among evaluable target lesions was 48\% (36\% CR and $12 \% \mathrm{PR}$ ). Lesions that received a high dose (i.e., $0.2 \mathrm{~mL}$ or higher) showed a $69 \%$ ORR, as compared to an ORR of $25 \%$ in smaller lesions that received a lower dosage. In support of the hypothesis of the systemic immune response by PV-10; an ORR of $27 \%$ was observed in noninjected lesions, and was more pronounced (44\%) in patients demonstrating a positive response in the injected lesions (43).

This was followed by an international, multicenter, openlabel, phase II clinical trial (NCT00521053) (44) in which 80 participants with stages III-IV melanoma with at least one cutaneous or subcutaneous lesion were enrolled. The primary aim was the best ORR. IT PV-10 was administered in up to 20 cutaneous/subcutaneous lesions, up to 4 times, over a 16-week period. The time of follow-up was 52 weeks. In the target lesions, ORR was $51 \%$ with $26 \% \mathrm{CR}$. At the end of the follow-up period, $8 \%$ of participants showed 
no evidence of disease. Responses in noninjected lesions were directly associated with response in injected lesions. A response $(\mathrm{CR} / \mathrm{PR})$ in injected lesions correlated with $56 \%$ $\mathrm{CR}$ and $6 \% \mathrm{PR}$ in noninjected lesions whereas no response in injected lesions was associated with only $6 \% \mathrm{CR}$ and $12 \% \mathrm{PR}$ in noninjected lesions. No grade 4 or $5 \mathrm{AEs}$ were observed. Most AEs were mild-moderate local/regional symptoms such as pain (80\%), edema (41\%), vesicles (39\%) and discoloration (31\%) (44).

A phase III, multicenter, open-label, randomized trial (NCT02288897) aiming to compare single-agent IT PV-10 with systemic chemotherapy (dacarbazine or temozolomide) or IT T-VEC, in patients with locally advanced, cutaneous malignant melanoma was terminated due to an inadequate rate of enrollment. Other studies are assessing the safety, tolerability, and pharmacokinetics of PV-10 as a single agent in patients with primary liver cancer or metastatic solid tumors to the liver (including melanoma) (NCT00986661), or in combination with pembrolizumab in advanced melanoma (NCT02557321).

\section{TLRs agonists}

TLRs are single-pass, membrane-spanning, noncatalytic receptors which recognize molecular patterns associated with pathogens. They are expressed on immune cells (APCs, T cells, B cells, natural killer cells), non-immune cells such as endothelial cells, epithelial cells, as well as on malignant cells (e.g., melanoma) (45). It has been estimated that most mammals have ten to fifteen types of TLR. Thirteen TLRs (TLR-1-TLR-13) have been discovered in humans and mice (45). TLR-1, $-2,-4,-5,-6$, and -10 are expressed on the cell surface, whereas TLR-3, $-7,-8$, and -9 are expressed on endosomes and ER (intracellular compartments) (46). TLRs play a pivotal role in the transition between innate and adaptive immune systems. Their primary function is to identify and bind with conserved pathogen-associated molecular patterns (PAMPs) expressed on a wide range of pathogens or damage-associated molecular patterns (DAMPs) released from dying or stressed cells. Once stimulated they can orchestrate both pro and anti-tumor responses $(45,46)$.

\section{TLR-9 agonists \\ $S D-101$}

SD-101 is a synthetic oligonucleotide with cytidinephospho-guanosine (CpG) motifs which bind to TLR9 , thereby activating plasmacytoid dendritic cells, causing upregulation of IFN $\alpha$ production and their maturation to APCs (47). Administered IT, as a monotherapy or in combination with anti-PD-1 therapy, it has shown promising activity in both pre-clinical and clinical studies (47). Results of IT SD-101 in combination with pembrolizumab from 22 patients from a multicenter phase Ib study (NCT02521870) in patients with metastatic melanoma have been reported. The majority of AEs were grade 1 and 2 including flu-like symptoms, localized redness, and pain at the injection site. Grade 3 and 4 AEs included chills, myalgia, and injection-site pain (14\% each). One patient developed an $\mathrm{AE}$ in the injected extremity leading to discontinuation of only SD-101. In anti-PD-1 naïve patients ORR was $78 \%$ (CR 22\%, PR 56\%), while in patients with prior anti-PD1 ORR was $15 \%$ (no CR, $15 \%$ PR). Injected tumor biopsies showed a significant increase in $\mathrm{CD} 8^{+} \mathrm{T}$ cells, natural killer cells, cytotoxic cells, dendritic cells, and $\mathrm{B}$ cells which were more pronounced in PD-1 naïve patients (47). However, the study was terminated as part of strategic restricting of the clinical oncology development programs and no further sponsoring of the development of SD-101.

\section{CMP-001}

CMP-001; another TLR-9 agonist, is an oligodeoxynucleotide CpG-A DNA, coated by a noninfectious virus-like particle (VLP). With a similar mechanism to SD-101, it activates plasmacytoid dendritic cells $(48,49)$. A phase Ib multicenter study (NCT02680184) of CMP-001 in combination with pembrolizumab has been conducted in patients with advanced melanoma. Preliminary results from 68 participants showed an acceptable safety profile with the majority of AEs as fever, nausea, vomiting, headache, hypotension, and rigors. The most common grade 3/4 AEs were hypotension in seven patients and anemia, chills, hypertension, and fever in two patients each. The ORR in weekly $(n=40)$ and every 3 -week $(n=13)$ cohorts was $22.5 \%$ and $7.7 \%$ respectively (49). Another phase $\mathrm{Ib}$, multicenter, clinical trial (NCT03084640) is examining alternative routes of CMP-001 administration in combination with pembrolizumab in patients with advanced melanoma. A phase 2 clinical trial (NCT03618641) is evaluating neoadjuvant IT CMP-001 in combination with nivolumab, in patients with stage IIIB-D melanoma. The primary outcome is the assessment of the major pathological response rate.

\section{IMO-2125 (Tilsotolimod)}

IMO-2125 is a synthetic oligodeoxynucleotide, that also activates TLR-9 (48). ILLUMINATE-101 (NCT03052205) is a phase Ib study of IT IMO-2125 in patients with refractory solid tumors including melanoma. Preliminary 
results from 54 patients showed no DLTs (50). The most common treatment-related toxicities were fever, fatigue, and chills. Tumor biopsies revealed an increase in IFN- $\gamma$ levels, enhanced expression of type $1 \mathrm{IFN}$, and multiple immune checkpoint pathways, and upregulation of MHC class I and II compared to pretreatment levels. Of 43 evaluable patients, 15 (35\%) has a SD (50). ILLUMINATE-204 (NCT02644967) is a phase I/II study evaluating the recommended dose of IMO-2125 in combination with ipilimumab or pembrolizumab in patients with metastatic melanoma. Of 62 PD-1 refractory patients, 52 received the recommended phase II dose of $8 \mathrm{mg}$ in combination with ipilimumab, and 49 were evaluable for efficacy (51). The median OS was 21 months, ORR was $22.4 \%$ and the median duration of response was 11.4 months. The DCR was $71.4 \%$. Overall, $48 \%$ [30/62] of patients experienced $\geq$ grade 3 toxicities including elevation in alanine aminotransferase (ALT), aspartate aminotransferase (AST), and colitis. There were no treatment-related discontinuations or deaths (51). ILLUMINATE-301 (NCT03445533) is a phase III, multi-center, open-label, randomized trial evaluating ipilimumab with and without IT IMO-2125 in patients with PD-1 refractory, advanced melanoma. Estimated enrollment is 454 patients with an estimated completion date of September 2021.

\section{AST-008 (cavrotolimod)}

AST-008 is a novel spherical nucleic acid configuration of a TLR-9 agonist in which the oligonucleotides are radially arranged and densely packed on the surface of liposomal nanoparticles (52). This is predicted to result in more nuclease stability and increased cellular uptake. In preclinical models, subcutaneous, IT, and IV administration has been shown to induce strong anti-tumor activity and enhance CPI activity (52). IT AST-008 is currently being studied in a phase $\mathrm{Ib} / \mathrm{II}$, multicenter, open-label clinical trial (NCT03684785) in combination with anti-PD-1 therapies in patients with advanced solid malignancies including melanoma.

\section{MGN1703 (lefitolimod)}

MGN1703, another TLR-9 agonist, is a double stem-loop immunomodulator with a short covalently closed dumbbellshaped conformation $(48,53)$. It consists of two loops with three CpG motifs each, linked together through a stable double-stranded linker (48). It's shown to be stable against cellular nuclease degradation with a better toxicity profile and immunostimulatory activity as compared to singlestranded CpG oligodeoxynucleotides $(48,53)$. In a phase I study in 28 patients with metastatic solid tumors, fatigue and prolonged activated partial thromboplastin time were the only two cases of drug-related grade 3 toxicities (53). Currently, a phase I clinical study (NCT02668770) is evaluating IT MGN1703 in combination with IV Ipilimumab in patients with advanced solid tumors, including melanoma.

\section{Other TLR agonists CV8102}

CV8102 is a 547 -nucleotide, uncapped single-stranded, non-coding RNA containing PolyU repeats complexed with a carrier consisting of disulfide-cross-linked cationic peptides with the sequence $\mathrm{CR}_{12} \mathrm{C}$ (54). It acts as a TLR7/8 and RIG-I (retinoic acid-inducible gene I) agonist to stimulate the innate and adaptive immune system $(54,55)$. IT CV8102 is currently under investigation as a monotherapy or in combination with anti-PD-1 therapy in a phase I, open-label, dose-escalation, and expansion study (NCT03291002), In patients with advanced solid tumors including melanoma. The preliminary results most common grade 1/2 AEs were fatigue, fever, chills, and headaches. Noted grade 3 treatment-related AEs were elevation in liver enzymes, abscess at the injection site, hypertension, and asymptomatic elevation of pancreatic enzymes. Objective responses in melanoma patients both in PD-1 naïve and refractory settings were noted (55).

\section{NKTR-262}

NKTR-262 is an IT administered TLR-7/8 agonist (56). In phase I/II REVEAL Trial (NCT03435640) NKTR262 is being evaluated in combination with NKTR-214 (a recombinant human IL-2) with or without nivolumab in patients with advanced solid malignancies including melanoma. Preliminary results from 11 patients treated with NKTR-262 with NKTR-214 combination showed transient flu-like symptoms as the most common grade $1 / 2$ AEs with no grade 3 or higher treatment-related AEs (57).

\section{LHC165}

LHC165 is a benzonapthyridine TLR-7 agonist that is adsorbed to aluminum hydroxide to allow for a slow release from the injection site (58). A phase I/1b, open-label, multicenter, dose-escalation, and dose-expansion clinical trial (NCT03301896) is evaluating the safety, tolerability, preliminary efficacy, and pharmacological properties of IT administered LHC165 as monotherapy or in combination with PDR001.

\section{G100}

G100 (GLA-SE) is an IT administered, synthetic TLR4 agonist, made from a glucopyranosyl lipid-A (GLA) 
prepared in stable oil-in-water emulsion (GLA-SE) (59). NCT02387125 was a phase I trial which aimed to have an exploratory analysis of IT G100 in the context of CMB305 [sequentially administered LV305 (a dendritic cell-targeting viral vector expressing the NY-ESO-1 gene) and G305 (NYESO-1 recombinant protein plus GLA-SE)] in patients with advanced malignancies expressing NY-ESO-1. The study failed to meet the efficacy objective.

\section{Intralesional $I L-2$}

Systemic IL-2 was approved by the FDA for metastatic melanoma treatment in 1998, however, treatment was limited by high rates of systemic side effects and low response rates (60). Intralesional IL-2 has shown promising results in terms of efficacy and safety in cancer management (61) but progress has been limited due to high cost and burdensome administration protocol (62). A phase I/II clinical trial (NCT03474497) is evaluating the safety and efficacy of the combination of IL-2 with radiotherapy and pembrolizumab in patients with PD-1 refractory advanced solid malignancies including metastatic melanoma. A phase III study (NCT03233828) is recruiting patients with suspected melanoma, or melanoma in situ and is randomizing to IT IL-2 vs. placebo to assess whether pretreatment of IL-2 on lesions is effective in generating an adaptive immune response, and whether that immune response may play a role in preventing disease metastasis. Another planned phase II/III clinical trial (NCT03928275) is randomizing patients with cutaneous metastatic melanoma to IT IL-2 with or without Bacillus Calmette Guerin (BCG).

\section{Daromun $($ L19IL2 + L19TNF)}

Daromun consists of recombinant fusion proteins of IL-2 and TNF- $\alpha$ fused to L19 monoclonal antibody (63). L19 antibody preferentially binds to extra-domain B of fibronectin, a molecule abundantly found in tumor blood vessels and a marker of tumor angiogenesis (63). This allows Daromun to accumulate and last longer in the injected lesions with a more sustained immunological effect as compared to non-targeted cytokines as demonstrated in preclinical models (63). In a phase II study IT L19IL2 and L19TNF were administered in patients with unresectable stage IIIC and IVM1a metastatic melanoma (64). Among 20 patients evaluable for response, the ORR was $55 \%$ including 1 patient with CR (5\%) and 10 (50\%) with PR.
The DCR of treated lesions was $80 \%$. Among the noninjected 13 lesions, 7 (54\%) had CR. Most AEs were grade 1 and 2 with exception of 2 grade 3 injection site reactions (64). Currently, neoadjuvant IT Daromun is being studied in open-label phase III clinical trials in patients with stages IIIB and C melanoma (NCT02938299 and NCT03567889).

\section{Hiltonol (poly-ICLC)}

Polyinosinic-polycytidylic acid-poly-l-lysine carboxymethylcellulose (poly-ICLC) or Hiltonol is a synthetic double-stranded RNA mimic of PAMP or "danger signal" that binds TLR-3, melanoma differentiationassociated protein 5 (MDA-5), and other pathogen receptors activating innate and adaptive immunity through induction of interferon, other cytokines and chemokines, APCs, T cells, and natural killer cells (65). In a pilot study, IT followed by IM Hiltonol was evaluated for safety in patients with advanced solid tumors including melanoma. Treatment was generally well-tolerated, and majority of AEs were grade 1 and 2 (mainly fatigue and injection site inflammation). One patient developed grade $3 \mathrm{AE}$ (aspiration pneumonia/pneumonitis) that was deemed as unlikely related to Hiltonol. Immunohistochemical analysis and RNA sequencing performed in patients who responded to treatment showed a significant increase in immune response (65). These encouraging results led to the progression to a phase II (NCT02423863) study evaluating IT and IM Hiltonol in patients with advanced solid tumors.

\section{IFx-Hu2.0}

IFx-Hu2.0 consists of a plasmid DNA encoding for streptococcal membrane protein Emm55 and a cationic polymer (66). An early phase I clinical trial (NCT03655756) has evaluated IT IFx-Hu2.0 in patients with stage III and IV, unresectable, cutaneous melanoma.

\section{Electroporation}

Electroporation is the process of administering brief electrical pulses directly to an accessible tumor that will result in inducing an electric field that exceeds the transmembrane potential of the plasma membrane leading to increased permeability of tumor-cell walls to certain molecules that under normal circumstances are unable to penetrate the cell membrane (67). Traditionally, 
electroporation has been used with chemotherapy (i.e., electrochemotherapy) with agents such as bleomycin and cisplatin, but it has also been used to include gene therapy and most recently calcium electroporation (67).

\section{Calcium electroporation}

Calcium electroporation utilizes a similar mechanism, that leads to internalization of supraphysiological amount of calcium, resulting in tumor necrosis due to sudden exhaustion of adenosine triphosphate (ATP) reserve (67). In a phase II, non-inferiority trial, evaluating calcium electroporation versus electrochemotherapy with bleomycin in patients with breast cancer and melanoma with cutaneous metastases; calcium electroporation showed a similar tumor response to electrochemotherapy $(72 \%$ vs. $84 \%, \mathrm{P}$ value $=0.5)(68)$. A randomized confirmatory, phase II trial (NCT03628417) is comparing IT calcium electroporation with electrochemotherapy with bleomycin in patients with cutaneous metastases of any histology.

\section{Plasmid interleukin-12 electroporation (pIL-12 EP)}

Plasmid IL-12 pUMVC3-hIL-12-NGVL3 [tavokinogene telseplasmid (tavo), pIL-12] encodes genes for p35 and p40 subunits of the heterodimeric human IL-12 protein which are separated by an internal ribosome entry site (69). A single cytomegalovirus promoter drives constitutive expression of the subunits. Once transfected with the plasmid, tumor cells express and secrete IL-12p70 protein inducing local and systemic immune responses (69). Electroporation pulses allow tavo to enter the cells and therefore only cells that are simultaneously exposed to both electroporation and tavo plasmid DNA are transfected, ensuring targeted delivery of the plasmid and local secretion of IL-12 (69). In a phase I trial of IT pIL-12 electroporation (NCT00323206) the major AE was pain at the electroporation site. Treatment was well tolerated with no DLTs. Results showed $10 \%$ CR, and $42 \%$ PR or SD. Biopsies obtained after electroporation showed a marked increase in IL-12 concentration, lymphocytes count and tumor necrosis compared to pre-treatment biopsies (70). In the phase II trial of tavo in patients with stage III/IV melanoma (NCT01502293), the ORR was $35.7 \%$ in the main study of which $17.9 \%$ were CR. Transcriptomic and immunohistochemistry analysis revealed not only upregulation of immune and co-stimulatory transcripts but also of pathways of adaptive immune resistance as demonstrated by an increase in PD-L1 expression by immunohistochemistry (71). Due to these results, two phase
II clinical trials ((NCT02493361 and NCT03132675) are currently evaluating IT pIL-12 EP in combination with IV anti-PD-1 therapy in patients with advanced melanoma.

\section{IT immunotherapy and chemotherapy}

\section{IT ipilimumab}

Ipilimumab is a fully human, $\operatorname{IgG1}$ monoclonal antibody directed against cytotoxic T-lymphocyte-associated antigen-4 (CTLA-4) (1). Since systemic ipilimumab administration is associated with severe AEs; IT ipilimumab was evaluated in combination with IT IL-2 in a phase I trial (NCT01672450) in patients with advanced-stage melanoma. There were no DLTs with $67 \%$ of patients experiencing local response in injected lesions, $89 \%$ abscopal response, and $40 \%$ ORR (72). IT Ipilimumab is currently being studied in a randomized phase I/II study (NCT02857569) which is comparing the combination of IT ipilimumab with IV nivolumab, to systemic ipilimumab and nivolumab in patients with metastatic melanoma.

\section{INT230-6}

INT230-6 is a formulation of cisplatin, vinblastine with an amphiphilic cell penetration enhancer in a fixed ratio which improves diffusion and dispersion into cancer cells once delivered intratumorally (73). Preliminary results from a phase I/II trial (NCT03058289) of INT230-6 in patients with advanced solid malignancies including melanoma reported no DLTs (73). The majority of AEs were local grade 1 and 2 symptoms (pain, infection, and blistering at the injection site). This trial is still ongoing with a total of nine cohorts, including cohorts with escalated INT230-6 dose, and a combination of INT230-6 with CPI.

\section{TTI-621 (SIRP $\alpha F c)$}

One of the mechanisms in which tumors evade host immune response is by expressing glycoprotein CD47 on its surface, which binds to signal-regulatory protein alpha $(\mathrm{SIRP} \alpha)$ expressed on the cell surface of macrophages present in the tumor microenvironment which blocks the phagocytic function of these cells $(74,75)$. CD47 is abundantly expressed by a wide variety of hematologic and solid malignant cells including melanoma $(74,75)$. TTI-621 $(\mathrm{SIRP} \alpha \mathrm{Fc})$ is a soluble recombinant protein consisting of the N-terminal V domain of human SIRP $\alpha$ (CD47 binding domain) fused to the $\mathrm{Fc}$ region of human immunoglobulin G1 (IgG1). TTI-621 acts by binding to CD47, thereby 
inhibiting the CD47-SIRP $\alpha$ signal, thereby blocking the inhibitory "do not eat" (antiphagocytic) signal to macrophages $(74,75)$. Due to promising preclinical results (74,75). a multicenter, phase I clinical trial (NCT02890368) of IT TTI-621 in refractory solid malignancies including melanoma as a monotherapy or in combination with other therapies has been conducted.

\section{CD-40 agonistic antibodies}

As a part of the tumor necrosis factor receptor superfamily (TNFRSF), activation of the CD40 receptor and its ligand plays an essential part in stimulating the humoral and cytotoxic immune responses. It is ubiquitously expressed on the cellular membrane of APCs (monocytes and dendritic cells), B lymphocytes, and on tumors of epithelial origin (76). ABBV-927, an agonistic monoclonal antibody against CD40 is being investigated in a phase I trial (NCT02988960) either as a single therapy (IT or IV) or in combination with IV ABBV-181 (an anti-PD-1 monoclonal antibody) in patients with solid tumors. APX005M (NCT02706353) is another intratumorally administered CD40 agonistic monoclonal antibody, undergoing phase I/II clinical trial in combination with pembrolizumab in patients with metastatic melanoma.

\section{Antimicrobial peptide LL37}

LL37, an endogenous 37 amino acid cationic peptide, is part of the cathelicidin family, that has antimicrobial activity against bacteria, viruses, fungi, and parasites. However, it also modulates a wide range of cellular functions including proliferation, invasion, apoptosis, cell cycle arrest, and cytokine release, and has both pro and anti-tumorigenic effects (77). It's anticancer potential stems from its role in enhancing human B lymphocytes and plasmacytoid dendritic cells' ability to recognize and bind to $\mathrm{CpG}$ oligodeoxynucleotide and subsequently leading to TLR9 activation (78). Other anticancer mechanisms have been discussed elsewhere (77). A phase I/II clinical trial (NCT02225366) has investigated LL37 as an IT therapy in melanoma patients.

\section{Dendritic cell therapy}

A phase I/II trial (NCT03325101) aims to evaluate the efficacy of dendritic cell therapy after cryosurgery in combination with pembrolizumab in patients with unresectable stage III-IV melanoma. Per available information patients undergo apheresis on day 1 or course 1 and receive pembrolizumab. Within 36 hours of receiving pembrolizumab patients undergo cryosurgery over 45 minutes on days 1 or 2 of courses 2 and 3 and after that receive IT autologous mature dendritic cells (on days 1 or 2 of courses 2 and 3 after cryosurgery).

\section{Polidocanol injection}

Polidocanol, is a sclerosing agent, composed of alkyl polyglycol ether of lauryl alcohol (79). That results in cell death by activating nitric oxide and calcium signaling pathways (79). INTRANS is a phase II clinical trial (NCT03754140) that is evaluating the efficacy of IT polidocanol injections in patients with in-transit cutaneous melanoma metastases.

\section{Conclusions}

The rapid progress in our understanding of the immune system and mechanisms by which melanoma evades the immune system has been matched by novel agents that are being translated from preclinical studies to clinical trials. Many melanoma patients present with cutaneous and subcutaneous lesions, locoregional disease, or intransit metastasis which provides a unique and attractive opportunity to investigate direct, IT delivery of these investigational agents and understand their mechanisms of action through sequential biopsies. So far T-VEC remains the only intralesional agent to be approved for the treatment of melanoma. In this review, we present the most comprehensive discussion of intralesional agents that have reached clinical trials along with their mechanisms of action. With the phenomenal success of anti-PD-1 therapies, most studies are adopting anti-PD-1 agents as the backbone of treatment and focusing the development in PD-1 refractory setting. Ongoing studies will further clarify the exact roles of these agents.

\section{Acknowledgments}

Funding: None.

\section{Footnote}

Provenance and Peer Review: This article was commissioned by the editorial office, Annals of Translational Medicine for 
the series "Cancer Immunotherapy: Recent Advances and Challenges". The article has undergone external peer review.

Conflicts of Interest: All authors have completed the ICMJE uniform disclosure form (available at http:// dx.doi.org/10.21037/atm-21-491). The series "Cancer Immunotherapy: Recent Advances and Challenges" was commissioned by the editorial office without any funding or sponsorship. US and MM served as the unpaid Guest Editors of the series. US serves as an unpaid editorial board member of Annals of Translational Medicine from May 2019 to Apr 2021. Dr. US reports consultancy/advisory board to Seattle Genetics. Dr. MM reports consultancy/advisory board for Blueprints Medicine, Immunocore, Amgen, Trieza, Array Biopharma, Biontech and Novartis. He also currently serves on Exicure Executive Steering Committee. Dr. YZ reports Advisory Board for BMS, Amgen, Roche Diagnostics, Novartis, Janssen, Eisai, Exelixis, Castle Bioscience, Array, Bayer, Pfizer, Clovis, EMD serono; institution clinical trial support from NewLink Genetics, Pfizer, Exelixis, and Eisai; Data Safety Monitoring Committee for Janssen Research and Development; consultant honorarium from Pfizer, Novartis. The authors have no other conflicts of interest to declare.

Ethical Statement: The authors are accountable for all aspects of the work in ensuring that questions related to the accuracy or integrity of any part of the work are appropriately investigated and resolved.

Open Access Statement: This is an Open Access article distributed in accordance with the Creative Commons Attribution-NonCommercial-NoDerivs 4.0 International License (CC BY-NC-ND 4.0), which permits the noncommercial replication and distribution of the article with the strict proviso that no changes or edits are made and the original work is properly cited (including links to both the formal publication through the relevant DOI and the license). See: https://creativecommons.org/licenses/by-nc-nd/4.0/.

\section{References}

1. Luther C, Swami U, Zhang J, et al. Advanced stage melanoma therapies: Detailing the present and exploring the future. Crit Rev Oncol Hematol 2019;133:99-111.

2. Korn EL, Liu PY, Lee SJ, et al. Meta-analysis of phase II cooperative group trials in metastatic stage IV melanoma to determine progression-free and overall survival benchmarks for future phase II trials. J Clin Oncol 2008;26:527-34.

3. Alexandrov LB, Nik-Zainal S, Wedge DC, et al. Signatures of mutational processes in human cancer. Nature 2013;500:415-21. Erratum in: Nature. 2013 Oct 10;502(7470):258. Imielinsk, Marcin [corrected to Imielinski, Marcin].

4. Davies H, Bignell GR, Cox C, et al. Mutations of the BRAF gene in human cancer. Nature 2002;417:949-54.

5. Dock G. The Influence of Complicating Diseases Upon Leukemia. The American Journal of the Medical Sciences (1827-1924) 1904;127:563.

6. Hammill AM, Conner J, Cripe TP. Oncolytic virotherapy reaches adolescence. Pediatr Blood Cancer 2010;55:1253-63.

7. Russell SJ, Peng KW, Bell JC. Oncolytic virotherapy. Nat Biotechnol 2012;30:658-70.

8. Liu BL, Robinson M, Han ZQ, et al. ICP34.5 deleted herpes simplex virus with enhanced oncolytic, immune stimulating, and anti-tumour properties. Gene Ther 2003;10:292-303.

9. Hu JC, Coffin RS, Davis CJ, et al. A phase I study of OncoVEXGM-CSF, a second-generation oncolytic herpes simplex virus expressing granulocyte macrophage colonystimulating factor. Clin Cancer Res 2006;12:6737-47.

10. Senzer NN, Kaufman HL, Amatruda T, et al. Phase II clinical trial of a granulocyte-macrophage colonystimulating factor-encoding, second-generation oncolytic herpesvirus in patients with unresectable metastatic melanoma. J Clin Oncol 2009;27:5763-71.

11. Andtbacka RH, Kaufman HL, Collichio F, et al. Talimogene Laherparepvec Improves Durable Response Rate in Patients with Advanced Melanoma. J Clin Oncol 2015;33:2780-8.

12. Puzanov I, Milhem MM, Minor D, et al. Talimogene Laherparepvec in Combination with Ipilimumab in Previously Untreated, Unresectable Stage IIIB-IV Melanoma. J Clin Oncol 2016;34:2619-26.

13. Chesney J, Puzanov I, Collichio F, et al. Randomized, Open-Label Phase II Study Evaluating the Efficacy and Safety of Talimogene Laherparepvec in Combination With Ipilimumab Versus Ipilimumab Alone in Patients With Advanced, Unresectable Melanoma. J Clin Oncol 2018;36:1658-67.

14. Ribas A, Dummer R, Puzanov I, et al. Oncolytic Virotherapy Promotes Intratumoral T Cell Infiltration and Improves Anti-PD-1 Immunotherapy. Cell 2017;170:1109- 
1119.e10. Erratum in: Cell. 2018 Aug 9;174(4):1031-1032. doi: 10.1016/j.cell.2018.07.035.

15. Andtbacka RHI, Dummer R, Gyorki DE, et al. Interim analysis of a randomized, open-label phase 2 study of talimogene laherparepvec (T-VEC) neoadjuvant treatment (neotx) plus surgery (surgx) vs surgx for resectable stage IIIB-IVM1a melanoma (MEL). J Clin Oncol 2018;36:abstr 9508.

16. Wei D, Xu J, Liu XY, et al. Fighting Cancer with Viruses: Oncolytic Virus Therapy in China. Hum Gene Ther 2018;29:151-9.

17. Cui C, Lian B, Chi Z, et al. OrienX010 oncolytic viral therapy in phase Ic trial of intralesional injection in liver metastases among patients with stage IV melanoma after standard treatment. J Clin Oncol 2017;35:e21013.

18. Eissa IR, Naoe Y, Bustos-Villalobos I, et al. Genomic Signature of the Natural Oncolytic Herpes Simplex Virus HF10 and Its Therapeutic Role in Preclinical and Clinical Trials. Front Oncol 2017;7:149.

19. Ferris RL, Gross ND, Nemunaitis JJ, et al. Phase I trial of intratumoral therapy using HF10, an oncolytic $\mathrm{HSV}-1$, demonstrates safety in $\mathrm{HSV}+/ \mathrm{HSV}$ - patients with refractory and superficial cancers. J Clin Oncol 2014;32:abstr 6082.

20. Andtbacka RHI, Ross MI, Agarwala SS, et al. Final results of a phase II multicenter trial of HF10, a replicationcompetent HSV-1 oncolytic virus, and ipilimumab combination treatment in patients with stage IIIBIV unresectable or metastatic melanoma. J Clin Oncol 2017;35:abstr 9510.

21. Andtbacka RHI, Ross MI, Agarwala SS, et al. Efficacy and genetic analysis for a phase II multicenter trial of HF10, a replication-competent HSV-1 oncolytic immunotherapy, and ipilimumab combination treatment in patients with stage IIIb-IV unresectable or metastatic melanoma. J Clin Oncol 2018;36:abstr 9541.

22. Nakayama T, Yamashita M, Suzuki T, et al. Immunological impact of canerpaturev (C-REV, formerly HF10), an oncolytic viral immunotherapy, with or without ipilimumab (Ipi) for advanced solid tumor patients (pts). J Clin Oncol 2019;37:abstr 2610.

23. Bradley S, Jakes AD, Harrington $\mathrm{K}$, et al. Applications of coxsackievirus A21 in oncology. Oncolytic Virother 2014;3:47-55.

24. Shafren D, Smithers BM, Formby M. A phase I, openlabel, cohort study of two doses of coxsackievirus A21 given intratumorally in stage IV melanoma. J Clin Oncol 2011;29:abstr 8573 .
25. Andtbacka RHI, Curti BD, Kaufman H, et al. Final data from CALM: A phase II study of Coxsackievirus A2 1 (CVA21) oncolytic virus immunotherapy in patients with advanced melanoma. J Clin Oncol 2015;33:abstr 9030.

26. Andtbacka RHI, Curti BD, Hallmeyer S, et al. Phase II calm extension study: Coxsackievirus A21 delivered intratumorally to patients with advanced melanoma induces immune-cell infiltration in the tumor microenvironment. J Immunother Cancer 2015;3: P343.

27. Curti B, Richards J, Hallmeyer S, et al. Abstract CT114: The MITCI (Phase 1b) study: A novel immunotherapy combination of intralesional Coxsackievirus A21 and systemic ipilimumab in advanced melanoma patients with or without previous immune checkpoint therapy treatment. Cancer Res 2017;77:abstr CT114.

28. Silk AW, Kaufman H, Gabrail N, et al. Abstract CT026: Phase 1 b study of intratumoral Coxsackievirus A21 (CVA21) and systemic pembrolizumab in advanced melanoma patients: Interim results of the CAPRA clinical trial. Cancer Res 2017;77:abstr CT026.

29. Walton RW, Brown MC, Sacco MT, et al. Engineered Oncolytic Poliovirus PVSRIPO Subverts MDA5Dependent Innate Immune Responses in Cancer Cells. J Virol 2018;92:e00879-18.

30. Pol JG, Atherton MJ, Bridle BW, et al. Development and applications of oncolytic Maraba virus vaccines. Oncolytic Virother 2018;7:117-28.

31. National Cancer Institute Drug Dictionary. Recombinant vesicular stomatitis virus-expressing interferon-beta and tyrosinase-related protein 1. Available online: https:// www.cancer.gov/publications/dictionaries/cancer-drug/ def/797080. Retrieved September 16, 2019.

32. Zarogoulidis P, Darwiche K, Sakkas A, et al. Suicide Gene Therapy for Cancer - Current Strategies. J Genet Syndr Gene Ther 2013;4:16849.

33. Aguilar LK, Guzik BW, Aguilar-Cordova E. Cytotoxic immunotherapy strategies for cancer: mechanisms and clinical development. J Cell Biochem 2011;112:1969-77.

34. Twitty CG, Diago OR, Hogan DJ, et al. Retroviral Replicating Vectors Deliver Cytosine Deaminase Leading to Targeted 5-Fluorouracil-Mediated Cytotoxicity in Multiple Human Cancer Types. Hum Gene Ther Methods 2016;27:17-31.

35. Yagiz K, Rodriguez-Aguirre ME, Lopez Espinoza F, et al. A Retroviral Replicating Vector Encoding Cytosine Deaminase and 5-FC Induces Immune Memory in Metastatic Colorectal Cancer Models. Mol Ther Oncolytics 2017;8:14-26. 
36. Kuryk L, Moller AW, Jaderberg M. Combination of immunogenic oncolytic adenovirus ONCOS-102 with anti-PD-1 pembrolizumab exhibits synergistic antitumor effect in humanized A2058 melanoma huNOG mouse model. Oncoimmunology 2018;8:e1532763.

37. Nemunaitis J, Tong AW, Nemunaitis M, et al. A phase I study of telomerase-specific replication competent oncolytic adenovirus (telomelysin) for various solid tumors. Mol Ther 2010;18:429-34.

38. Motwani M, Pesiridis S, Fitzgerald KA. DNA sensing by the cGAS-STING pathway in health and disease. Nat Rev Genet 2019;20:657-74.

39. Li A, Yi M, Qin S, et al. Activating cGAS-STING pathway for the optimal effect of cancer immunotherapy. J Hematol Oncol 2019;12:35.

40. Corrales L, Glickman LH, McWhirter SM, et al. Direct Activation of STING in the Tumor Microenvironment Leads to Potent and Systemic Tumor Regression and Immunity. Cell Rep 2015;11:1018-30.

41. Harrington KJ, Brody J, Ingham M, et al. Preliminary results of the first-in-human (FIH) study of MK-1454, an agonist of stimulator of interferon genes (STING), as monotherapy or in combination with pembrolizumab (pembro) in patients with advanced solid tumors or lymphomas. Ann Oncol 2018;29:viii712.

42. Toomey P, Kodumudi K, Weber A, et al. Intralesional injection of rose bengal induces a systemic tumor-specific immune response in murine models of melanoma and breast cancer. PLoS One 2013;8:e68561.

43. Thompson JF, Hersey P, Wachter E. Chemoablation of metastatic melanoma using intralesional Rose Bengal. Melanoma Res 2008;18:405-11.

44. Thompson JF, Agarwala SS, Smithers BM, et al. Phase 2 Study of Intralesional PV-10 in Refractory Metastatic Melanoma. Ann Surg Oncol 2015;22:2135-42.

45. Holldack J. Toll-like receptors as therapeutic targets for cancer. Drug Discov Today 2014;19:379-82.

46. Kaczanowska S, Joseph AM, Davila E. TLR agonists: our best frenemy in cancer immunotherapy. J Leukoc Biol 2013;93:847-63.

47. Ribas A, Medina T, Kummar S, et al. SD-101 in Combination with Pembrolizumab in Advanced Melanoma: Results of a Phase Ib, Multicenter Study. Cancer Discov 2018;8:1250-7.

48. Adamus T, Kortylewski M. The revival of CpG oligonucleotide-based cancer immunotherapies. Contemp Oncol (Pozn) 2018;22:56-60.

49. Milhem M, Gonzales R, Medina T, et al. Abstract CT144:
Intratumoral toll-like receptor 9 (TLR9) agonist, CMP001, in combination with pembrolizumab can reverse resistance to $\mathrm{PD}-1$ inhibition in a phase $\mathrm{Ib}$ trial in subjects with advanced melanoma. Cancer Res 2018;78:CT144.

50. Babiker H, Borazanci E, Subbiah V, et al. 1195P - Safety, efficacy, and immune effects of intratumoral tilsotolimod in patients with refractory solid tumours: Updated results from ILLUMINATE-101. Ann Oncol 2019;30:v487.

51. Haymaker C, Andtbacka RHI, Johnson DB, et al. 1083MO Final results from ILLUMINATE-204, a phase I/II trial of intratumoral tilsotolimod in combination with ipilimumab in PD-1 inhibitor refractory advanced melanoma. Ann Oncol 2020;31:S736.

52. Kandimalla ER, Nallagatla S, Anderson BR, et al. Abstract A136: AST-008, a novel TLR9 agonist SNA, induces abscopal antitumor effects in mouse tumor models. Cancer Immunol Res 2019;7:abstr A136.

53. Weihrauch MR, Richly H, von Bergwelt-Baildon MS, et al. Phase I clinical study of the toll-like receptor 9 agonist MGN1703 in patients with metastatic solid tumours. Eur J Cancer 2015;51:146-56.

54. Ziegler A, Soldner C, Lienenklaus S, et al. A New RNA-Based Adjuvant Enhances Virus-Specific Vaccine Responses by Locally Triggering TLR- and RLHDependent Effects. J Immunol 2017;198:1595-605.

55. Eigentler T, Bauernfeind FG, Becker JC, et al. A phase I dose-escalation and expansion study of intratumoral CV8102 as single-agent or in combination with anti-PD-1 antibodies in patients with advanced solid tumors. J Clin Oncol 2020;38:abstr 3096.

56. Kivimae S, Hennessy M, Pena R, et al. Abstract 3755 : Comprehensive antitumor immune activation by a novel TLR7/8 targeting agent NKTR-262 combined with CD122-biased immunostimulatory cytokine NKTR-214. Cancer Res 2018;78:abstr 3755.

57. Diab A, Marcondes M, Kotzin B, et al. Phase Ib: Preliminary clinical activity and immune activation for NKTR-262 [TLR 7/8 agonist] plus NKTR-214 [CD122biased agonist] in patients (pts) with locally advanced or metastatic solid tumors (REVEAL Phase Ib/II Trial). J Clin Oncol 2019;37:abstr 26.

58. Deane JA, Cortez GA, Li C, et al. Abstract 4128: Identification and characterization of LHC165, a TLR7 agonist designed for localized intratumoral therapies. Cancer Res 2019;79:abstr 4128.

59. Bhatia S, Miller NJ, Lu H, et al. Intratumoral G100, a TLR4 Agonist, Induces Antitumor Immune Responses and Tumor Regression in Patients with Merkel Cell 
Carcinoma. Clin Cancer Res 2019;25:1185-95.

60. Jiang T, Zhou C, Ren S. Role of IL-2 in cancer immunotherapy. Oncoimmunology 2016;5:e1163462.

61. Boyd KU, Wehrli BM, Temple CL. Intra-lesional interleukin-2 for the treatment of in-transit melanoma. J Surg Oncol 2011;104:711-7.

62. Sloot S, Rashid OM, Sarnaik AA, et al. Developments in Intralesional Therapy for Metastatic Melanoma. Cancer Control 2016;23:12-20.

63. Miura JT, Zager JS. Neo-DREAM study investigating Daromun for the treatment of clinical stage IIIB/C melanoma. Future Oncol 2019;15:3665-74.

64. Danielli R, Patuzzo R, Di Giacomo AM, et al. Intralesional administration of L19-IL2/L19-TNF in stage III or stage IVM1a melanoma patients: results of a phase II study. Cancer Immunol Immunother 2015;64:999-1009.

65. Kyi C, Roudko V, Sabado R, et al. Therapeutic Immune Modulation against Solid Cancers with Intratumoral PolyICLC: A Pilot Trial. Clin Cancer Res 2018;24:4937-48.

66. Markowitz J, Kodumudi KN, Aquino DBD, et al. Abstract CT119: Trial in progress: First in human Phase I study using a plasmid DNA coding for Emm55 streptococcal antigen (IFx-Hu2.0) in patients with unresectable stage III or stage IV cutaneous melanoma. Cancer Res 2019;79:abstr CT119.

67. Hoejholt KL, Muzic T, Jensen SD, et al. Calcium electroporation and electrochemotherapy for cancer treatment: Importance of cell membrane composition investigated by lipidomics, calorimetry and in vitro efficacy. Sci Rep 2019;9:4758.

68. Falk H, Matthiessen LW, Wooler G, et al. Calcium electroporation for treatment of cutaneous metastases; a randomized double-blinded phase II study, comparing the effect of calcium electroporation with electrochemotherapy. Acta Oncol 2018;57:311-9.

69. Canton DA, Shirley S, Wright J, et al. Melanoma treatment with intratumoral electroporation of

Cite this article as: Zawit M, Swami U, Awada H, Arnouk J, Milhem M, Zakharia Y. Current status of intralesional agents in treatment of malignant melanoma. Ann Transl Med 2021;9(12):1038. doi: 10.21037/atm-21-491 tavokinogene telseplasmid (pIL-12, tavokinogene telseplasmid). Immunotherapy 2017;9:1309-21.

70. Daud AI, DeConti RC, Andrews S, et al. Phase I trial of interleukin-12 plasmid electroporation in patients with metastatic melanoma. J Clin Oncol 2008;26:5896-903.

71. Algazi A, Bhatia S, Agarwala S, et al. Intratumoral delivery of tavokinogene telseplasmid yields systemic immune responses in metastatic melanoma patients. Ann Oncol 2020;31:532-40.

72. Ray A, Williams MA, Meek SM, et al. A phase I study of intratumoral ipilimumab and interleukin-2 in patients with advanced melanoma. Oncotarget 2016;7:64390-9.

73. El-Khoeuiry A, Siu LL, Azad N, et al. Phase I/II evaluation of intratumoral INT230-6 for the treatment of solid tumors. Ann Oncol 2018;29:viii413.

74. Petrova PS, Viller NN, Wong M, et al. TTI-621 (SIRPalphaFc): A CD47-Blocking Innate Immune Checkpoint Inhibitor with Broad Antitumor Activity and Minimal Erythrocyte Binding. Clin Cancer Res 2017;23:1068-79.

75. Lin GHY, Chai V, Lee V, et al. TTI-621 (SIRPalphaFc), a CD47-blocking cancer immunotherapeutic, triggers phagocytosis of lymphoma cells by multiple polarized macrophage subsets. PLoS One 2017;12:e0187262.

76. Johnson P, Challis R, Chowdhury F, et al. Clinical and biological effects of an agonist anti-CD40 antibody: a Cancer Research UK phase I study. Clin Cancer Res 2015;21:1321-8.

77. Chen X, Zou X, Qi G, et al. Roles and Mechanisms of Human Cathelicidin LL-37 in Cancer. Cell Physiol Biochem 2018;47:1060-73.

78. Hurtado P, Peh CA. LL-37 promotes rapid sensing of CpG oligodeoxynucleotides by B lymphocytes and plasmacytoid dendritic cells. J Immunol 2010;184:1425-35.

79. Eckmann DM. Polidocanol for endovenous microfoam sclerosant therapy. Expert Opin Investig Drugs 2009;18:1919-27. 